\title{
MOBILIDADE DE NITRATO EM COLUNAS DE SOLO SOB CONDIÇÕES DE ESCOAMENTO NÃO PERMANENTE ${ }^{1}$
}

\author{
Silvana Nunes da Costa ${ }^{2}$, Mauro Aparecido Martinez ${ }^{3}$, Antônio Teixeira de Matos ${ }^{4}$ \\ \& Victor Brasil Nunes Ramos ${ }^{5}$
}

\begin{abstract}
RESUMO
Um estudo de deslocamento de nitrato no solo, aplicado com a água de irrigação e em duas concentrações, sob condições de escoamento não permanente, foi realizado em colunas de lixiviação, preenchidas com terra fina seca ao ar, de um material de solo classificado como Latossolo Vermelho Amarelo (LVa; horizonte B). Determinaram-se os perfis de umidade e de concentração de distribuição de nitrato em quatro diferentes tempos, ou seja, 4, 24, 72 e 168 h, após início da aplicação. Os resultados dos perfis de distribuição de umidade mostraram que o nitrato apresentou picos de concentração à frente de umedecimento do solo. A aplicação de nitrato em maiores concentrações proporcionou a obtenção de picos de maior magnitude; entretanto, pouco alterou o perfil de distribuição do ânion no solo.
\end{abstract}

Palavras-chave: nitrato, deslocamento miscível, escoamento não-permanente

\section{NITRATE DISPLACEMENT IN SOIL COLUMNS UNDER UNSTEADY FLOW CONDITIONS}

\begin{abstract}
A study was conducted on nitrate displacement in soil applied at two different concentrations with the irrigation water, under unsteady conditions, using leaching columns filled with air-dried, sieved soil from a material classified as Oxisol (LVa; horizon B). The moisture and nitrate concentration patterns were determined at four different times, 4, 24, 72 and $168 \mathrm{~h}$ after application. The results of the soil moisture and nitrate distribution patterns behaved according to nitrate concentrations applied to the column's superior and inferior extremities.
\end{abstract}

Key words: nitrate, miscible displacement, unsteady flow

\section{INTRODUÇÃO}

$\mathrm{Na}$ agricultura moderna, grandes quantidades de substâncias químicas são distribuídas sobre a superfície do solo, na forma de fertilizantes, adubos orgânicos, inseticidas e herbicidas, e até mesmo resíduos diversos. Esses insumos, quando aplicados acima da capacidade-suporte do solo, podem liberar íons e compostos tóxicos ou não, que poderão poluir o solo e as águas subterrâneas. Os íons disponibilizados na solução do solo podem ser adsorvidos ao solo, absorvidos pelas plantas ou lixiviados das camadas superficiais do solo. Segundo Edwards et al. (1972) alguns íons, quando disponibilizados na

\footnotetext{
${ }^{1}$ Parte da tese doutorado do primeiro autor, pesquisa parcialmente financiada pela FAPEMIG/CNPq

${ }^{2}$ Pesquisadora Doutora, Bolsista do CNPq, DEAg/CCT/UFPB, Campina Grande, PB, Av. Aprígio Veloso 882, CEP 58109 - 970, Fone: (083) 310 1285, E-mail: silvana@ deag.ufpb.br

${ }^{3}$ Professor Titular, PhD, Bolsista do CNPq, DEA/UFV, Av. P. H. Rolfs, Viçosa, MG, CEP 36571 - 000, Fone: (031) 8991910

${ }^{4}$ Professor Adjunto, DS, Bolsista do CNPq, DEA/UFV, Fone: (031) 8991420

${ }^{5}$ M.Sc. Engenharia Agrícola, DEA/UFV, Fone: (075) 2521065
} 
solução do solo, são rapidamente deslocados no perfil, como é o caso do nitrogênio que, na forma de íons de nitrato, é altamente solúvel em água e apresenta grande mobilidade no solo. O nitrato, por ser um ânion, não é retido em solos cuja predominância de cargas seja negativa apresentando, por isso, grande potencial de lixiviação, o que pode causar sérios problemas de poluição às águas subterrâneas.

A aplicação de quantidades excessivas de fertilizantes nitrogenados tem sido freqüentemente sugerida como a principal fonte de contaminação de águas subterrâneas (Spalding et al. 1982).

Segundo Newbould (1989) o consumo de fertilizantes nitrogenados tem aumentado em escala global, sendo as estimativas de consumo de 111 - 134 milhões de toneladas a serem consumidas no ano 2000. As fontes de nitrogênio rapidamente disponíveis são sais inorgânicos de amônio, nitrato e uréia.

Para prevenir e minimizar a poluição de águas subterrâneas com nitrato, é necessário um controle das taxas de nitrogênio aplicadas anualmente ao solo, além da compreensão dos fatores intervenientes na mobilidade do ânion no solo. As formas nítricas, quando aplicadas diretamente na formulação de fertilizantes ou produzidas por nitrificação do amônio, são rapidamente solúveis na solução do solo. Esta alta solubilidade e a fraca interação com a matriz do solo possibilitam que o ânion acompanhe a frente de umedecimento da água no solo; assim, o nitrato tanto pode movimentar para baixo, sob condições de excessiva precipitação ou irrigação, quanto para cima, por capilaridade, durante estações extremamente secas (Muchovej \& Rechcigl, 1994). Entretanto, em solos positivamente carregados, os quais têm capacidade de troca aniônica não desprezível, o nitrato pode ser adsorvido. A textura do solo e o conteúdo de matéria orgânica têm maior influência nas perdas por lixiviação. Solos de textura mais grosseira e de baixo conteúdo de matéria orgânica tendem a permitir maiores perdas de nitrato por lixiviação, enquanto as menores perdas devem ocorrer em solos argilosos (Muchovej \& Rechcigl, 1994). Em geral, para os mesmos autores, perdas de nitrato por lixiviação em solos arenosos são mínimas quando o nitrogênio é aplicado em taxas menores que $200 \mathrm{~kg} \mathrm{ha}^{-1} \mathrm{ano}^{-1} \mathrm{e}$ aumenta rapidamente em taxas maiores. Nesses solos, os limites de potabilidade $\left(10 \mathrm{mg} \mathrm{L}^{-1}\right) \mathrm{da}$ água subterrânea foram suplantados para taxas de aplicação entre 350 e $400 \mathrm{~kg} \mathrm{ha}^{-1} \mathrm{ano}^{-1} \mathrm{em}$ áreas cultivadas com pastos.

Exner et al. (1991) verificaram movimento profundo de nitrato, em diferentes taxas de aplicação, sob condição de irrigação, cujos resultados indicaram que $95 \%$ do nitrato aplicado foram lixiviados para baixo da zona radicular da cultura e as concentrações médias de nitrato no pulso variaram de 34 a $70 \mathrm{mg} \mathrm{L}^{-1}$ de nitrato.

Na Europa e nos Estados Unidos, áreas com concentrações de nitrato acima de $50 \mathrm{mg} \mathrm{L}^{-1}$ podem ser consideradas potencialmente poluídas (Vanclooster et al., 1994). Altos concentrações de nitrato na água são problemáticas para potabilidade pois, no corpo humano, o nitrato tem o poder de reduzir a capacidade de transporte de oxigênio do corpo (Owens, 1994). Hubbard et al. (1991) afirmam que, mesmo quando esses valores estão acima de $10 \mathrm{mg} \mathrm{L}^{-1}$, já provocam problemas de saúde em crianças; desta forma, é importante que se disponha de informações a respeito dos fenômenos físicos e químicos que ocorrem durante a lixiviação, trocas iônicas e adsorção desses íons, favorecendo a compreensão de como ocorre sua movimentação no solo (Nielsen \& Biggar, 1961; van Genuchten $\&$ Wierenga, 1976). Assim, este trabalho tem como objetivo avaliar o deslocamento vertical de nitrato em colunas de solo, aplicado por meio da água de irrigação, sob condição de escoamento não permanente.

\section{MATERIAL E MÉTODOS}

Este trabalho foi conduzido no Laboratório de Água e Solo do Departamento de Engenharia Agrícola da Universidade Federal de Viçosa, em colunas de $1 \mathrm{~m}$ de comprimento, constituídas por anéis de tubos de PVC rígido, de $15 \mathrm{~cm}$ de diâmetro e $10 \mathrm{~cm}$ de comprimento. O solo utilizado foi classificado como Latossolo Vermelho-Amarelo, álico - LVa; horizonte B e suas características físicas e químicas estão apresentadas nas Tabelas 1 e 2, respectivamente. Os valores de teor de fósforo e potássio foram determinados através de extrações com Mehlich, os de alumínio e cálcio através de extrações com cloreto de potássio e o alumínio mais cálcio extraídos com $\mathrm{Ca}(\mathrm{OAc})_{2}, 0,5 \mathrm{~mol} \mathrm{~L}^{-1} \mathrm{com} \mathrm{pH} \mathrm{7,0.}$

$\mathrm{O}$ enchimento das colunas, iniciado pela base, foi feito com material de solo devidamente homogeneizado, o qual foi submetido a uma leve compactação, resultando em uma densidade do solo de $1,0 \mathrm{~g} \mathrm{~cm}^{-3}$, a qual foi determinada pelo método da proveta. A densidade de partículas foi determinada pelo método do balão volumétrico tendo-se obtido o valor médio foi de $2,69 \mathrm{~g} \mathrm{~cm}^{-3}$.

Como fonte de nitrato, utilizaram-se soluções de nitrato de cálcio, $\mathrm{Ca}\left(\mathrm{NO}_{3}\right)_{2} \cdot 4 \mathrm{H}_{2} \mathrm{O}$, aplicadas por meio da água de irrigação, nas concentrações de $830 \mathrm{mg} \mathrm{mL}^{-1} \mathrm{e}$ $1.250 \mathrm{mg} \mathrm{mL}^{-1}$ correspondentes a 80 e $120 \mathrm{~kg} \mathrm{ha}^{-1} \mathrm{de}$ nitrogênio, respectivamente.

Para cada concentração de solução aplicada foram montadas quatro colunas, correspondentes aos tempos de coleta de 4, 24, 72 e 168 h após início da aplicação, totalizando oito colunas.

O solo situado na base das colunas foi mantido saturado todo o período de percolação da solução, por meio de um sistema de reservatórios para controle do nível freático. Frascos de Mariotte foram utilizados, a fim de se estabelecer o nível freático na base das colunas, no momento em que se iniciou a aplicação da solução na parte de cima das colunas; este procedimento foi adotado com o objetivo de simular uma situação prática na qual se consideram condições de contorno do tipo fluxo e do tipo potencial constante, nas partes superior e inferior da região de estudo, respectivamente como, por exemplo, o caso de uma fertirrigação em uma área onde o lençol freático é raso.

A lâmina utilizada para as duas situações foi de $109,5 \mathrm{~mm}$, aplicada na extremidade superior das colunas, a partir de gotejadores desenvolvidos para esta finalidade. Frascos de Mariotte foram também utilizados, com o objetivo de se manter uma lâmina de água constante no reservatório sobre os gotejadores, a qual era responsável pela vazão constante nos mesmos durante o tempo de aplicação da solução. O tempo médio de aplicação da lâmina de irrigação foi de $2,5 \mathrm{~h}$, para as oito colunas, tempo suficiente para que a frente de molhamento atingisse, em média, a profundidade de $35 \mathrm{~cm}$ em todas as colunas e o escoamento se mantivesse em condições de não saturação. 
Tabela 1. Características físico-hídricas do material de solo utilizado nas colunas de lixiviação

\begin{tabular}{|c|c|c|c|c|c|c|c|}
\hline \multicolumn{3}{|c|}{$\begin{array}{c}\text { Textura } \\
\left(\text { dag kg }^{-1}\right)\end{array}$} & \multicolumn{2}{|c|}{$\begin{array}{c}\text { Densidade } \\
\left(\mathrm{g} \mathrm{cm}^{-3}\right)\end{array}$} & \multirow[t]{2}{*}{$\begin{array}{c}\text { Porosidade } \\
\left(\mathrm{cm}^{3} \mathrm{~cm}^{-3}\right)\end{array}$} & \multirow[t]{2}{*}{$\begin{array}{c}\theta_{\text {inicial }} \\
\left(\mathrm{cm}^{3} \mathrm{~cm}^{-3}\right)\end{array}$} & \multirow[t]{2}{*}{$\begin{array}{c}\text { Ko } \\
\left(\mathrm{cm} \mathrm{h}^{-1}\right)\end{array}$} \\
\hline Argila & Silte & Areia & Solo & Partículas & & & \\
\hline 69,2 & 7,2 & 23,6 & 1,01 & 2,69 & 0,6245 & 0,58 & 5,43 \\
\hline
\end{tabular}

Tabela 2. Características químicas do solo utilizado

\begin{tabular}{|c|c|c|c|c|c|c|c|c|c|c|c|c|}
\hline \multirow{3}{*}{$\begin{array}{l}\text { Carbono } \\
\text { dag kg }^{-1}\end{array}$} & \multicolumn{2}{|c|}{$\mathrm{pH}$} & $\mathrm{P}$ & $\mathrm{K}$ & $\mathrm{Al}$ & $\mathrm{Ca}$ & $\mathrm{H}+\mathrm{Al}$ & SB & $\mathrm{CTC}_{\text {efe }}$ & V & $\mathrm{m}$ & Nitrato \\
\hline & $\mathrm{KCl}$ & Água & \multicolumn{2}{|c|}{$\mathrm{mg} \mathrm{dm}^{-3}$} & \multicolumn{5}{|c|}{$\mathrm{cmol}_{\mathrm{c}} \mathrm{dm}^{-3}$} & \multicolumn{2}{|c|}{$\%$} & $\mu \mathrm{g} \mathrm{mL}^{-1}$ \\
\hline & 4,4 & 4,5 & 0,3 & 4,0 & 0,5 & 0,2 & 3,0 & 0,59 & 1,09 & 16,5 & 45,7 & 120 \\
\hline
\end{tabular}

Na superfície das colunas foi colocada uma camada de lã de vidro de pequena espessura, para favorecer melhor uniformidade de distribuição de água; decorrido o tempo de aplicação da solução, as colunas foram cobertas com plástico para evitar evaporação na sua superfície durante o período de distribuição de umidade.

As amostras de solo foram coletadas, em cada coluna, a 5 $\mathrm{cm}$ de profundidade, e a cada $10 \mathrm{~cm}$ até a profundidade de $95 \mathrm{~cm}$, a partir da superfície superior e, finalmente, na parte inferior das colunas. Foram determinadas a umidade volumétrica pelo método gravimétrico e a concentração de nitrato por colorimetria, pela reação de Griess-Llosvay, desenvolvido para um sistema de fluxo de injeção automática, segundo adaptação de Alves (1992). Por este método, de cada amostra de solo, após homogeneização, retiravam-se $5 \mathrm{~g}$, que foram colocados em frasco erlenmeyer com capacidade $\mathrm{p}$ ara $250 \mathrm{~mL}$, ao qual se adicionavam $50 \mathrm{~mL}$ de uma solução de $\mathrm{KCl} 2 \mathrm{~mol} \mathrm{~L}^{-1}$, para extração da solução do solo. As concentrações foram calculadas em unidades de massa de nitrato por unidade de volume de solução do solo e de volume de solo.

\section{RESULTADOS E DISCUSSÃO}

Na Figura 1 estão apresentados os perfis de distribuição de umidade ao longo das colunas. Verifica-se que esta distribuição ocorreu em cada tempo, de forma semelhante nas diferentes colunas, indicando que houve uniformidade na montagem das colunas e na aplicação da lâmina de irrigação. Na parte inferior das colunas, onde o nível freático foi controlado, o efeito da ascensão capilar também foi semelhante em todos os tempos analisados e o solo permaneceu próximo à saturação na camada de 90 a $100 \mathrm{~cm}$ de profundidade.

Na Figura 2 estão apresentados os perfis de distribuição de concentração de nitrato no solo ao longo das colunas. Pode-se observar que, de 5 a $10 \mathrm{~cm}$ de profundidade, a concentração de nitrato encontrava-se inicialmente alta; em seguida, nota-se redução brusca neste valor, nas concentrações entre as profundidades de 10 a $20 \mathrm{~cm}$ (Fig. 2). Observa-se certa defasagem entre a frente de umedecimento e a frente de contaminação de nitrato no solo, estando a frente de contaminação atrasada em relação à de umedecimento. Este atraso na contaminação do nitrato deve-se à presença de cargas positivas no solo estudado, o que pode ser reconhecido pela pequena diferença obtida entre as determinações do $\mathrm{pH}$ em $\mathrm{KCl}$ e em água (Tabela 2). A presença de cargas positivas favorece a adsorção do nitrato junto à fase sólida do solo. Observou-se que, a partir de cerca de $40 \mathrm{~cm}$ de profundidade, os valores de concentração de nitrato no solo começaram a decrescer, assumindo valores mais ou menos semelhantes e próximos aos da concentração inicial de nitrato no solo, antes da aplicação da solução.

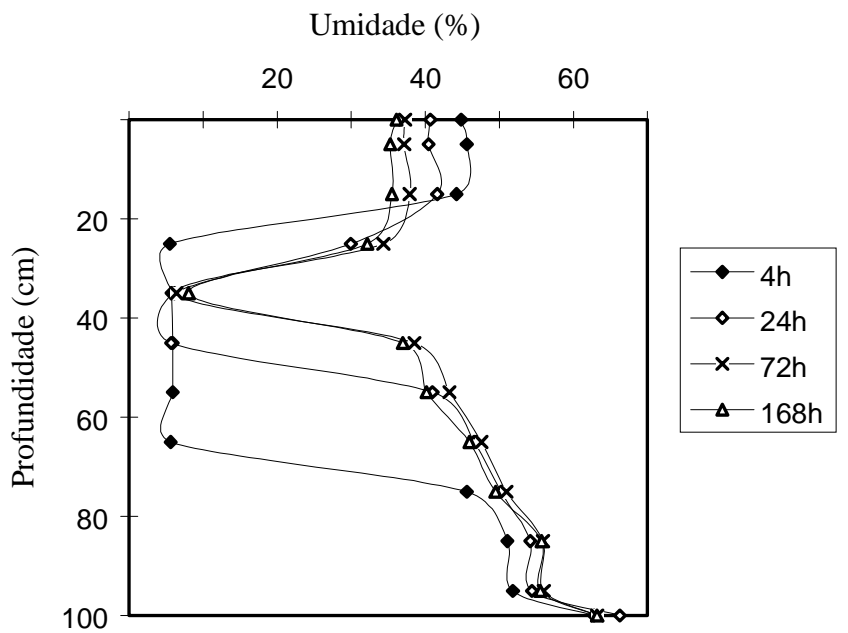

(A)

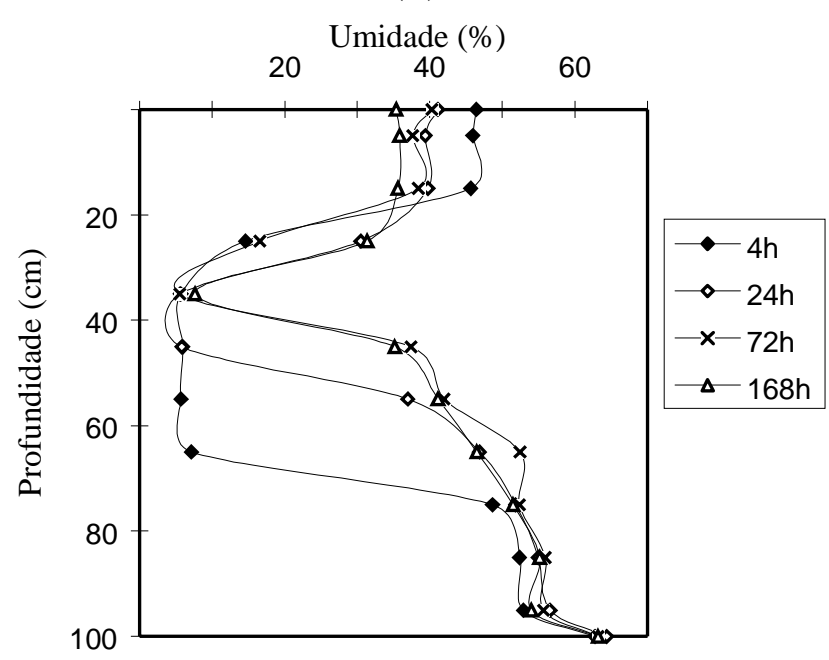

(B)

Figura 1. Distribuição de umidade no solo sob diferentes concentrações de aplicação de nitrato na solução (A) $830 \mathrm{mg}$ $\mathrm{mL}^{-1} \mathrm{e}$ (B) $1.250 \mathrm{mg} \mathrm{mL}^{-1}$ 


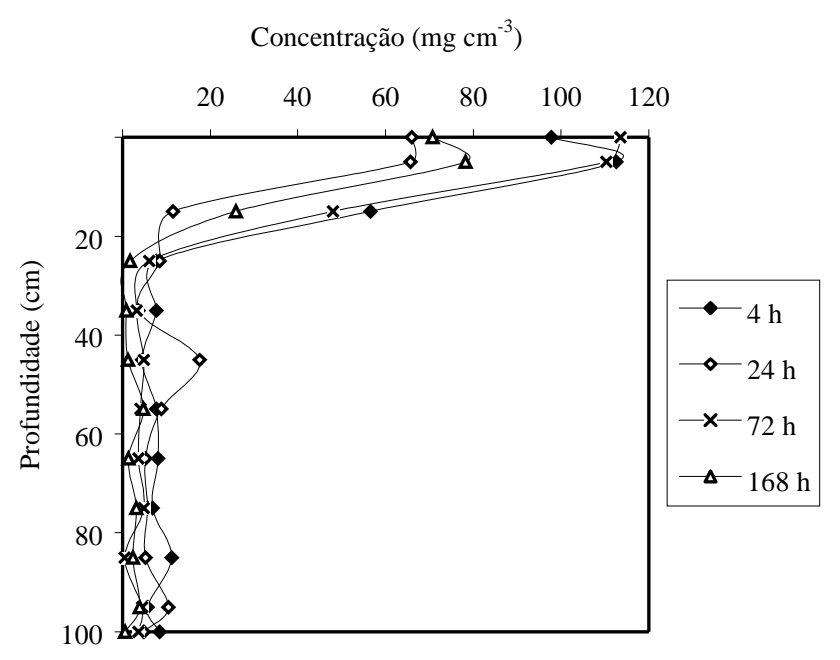

(A) $830 \mathrm{mg} \mathrm{mL}^{-1}$

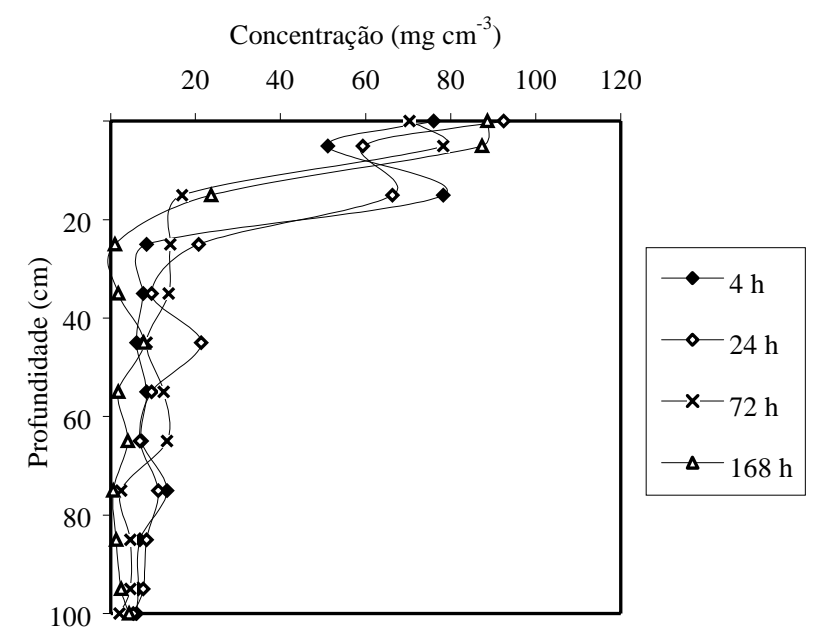

(B) $1.250 \mathrm{mg} \mathrm{mL}^{-1}$

Figura 2. Concentração de nitrato no solo ao longo de seu perfil, em períodos distintos de tempo após sua aplicação, nas concentrações de (A) $830 \mathrm{mg} \mathrm{mL}^{-1}$; (B) $1.250 \mathrm{mg} \mathrm{mL}^{-1}$, na superfície superior das colunas.

Embora a solução aplicada de $1.250 \mathrm{mg} \mathrm{mL}^{-1}$ de nitrato tenha proporcionado o aparecimento de um pico de concentração um pouco mais profundo, não foram observadas grandes diferenças na concentração de nitrato no perfil do solo das colunas. Acredita-se que o baixo conteúdo de água no solo, a partir da profundidade de $25 \mathrm{~cm}$, tenha sido de grande importância para que isso ocorresse. Com a ausência do transporte por fluxo de massa, o transporte difusivo passou a ser o único capaz de proporcionar o deslocamento do nitrato. A difusão do nitrato em ambiente de baixa disponibilidade de água tornam-se difícil.

Analisando-se a concentração de nitrato na solução do solo (Figura 3) observou-se que, para o tempo de 4 horas, embora a frente de umedecimento estivesse ainda a cerca de $35 \mathrm{~cm}$ de profundidade, o nitrato já atingira cerca de $70 \mathrm{~cm}$; entretanto, esta distribuição do nitrato na solução do solo não deve ser creditada ao avanço da frente de contaminação e, sim, à concentração original do ânion, em decorrência da menor disponibilidade de água no solo de determinadas camadas da coluna.

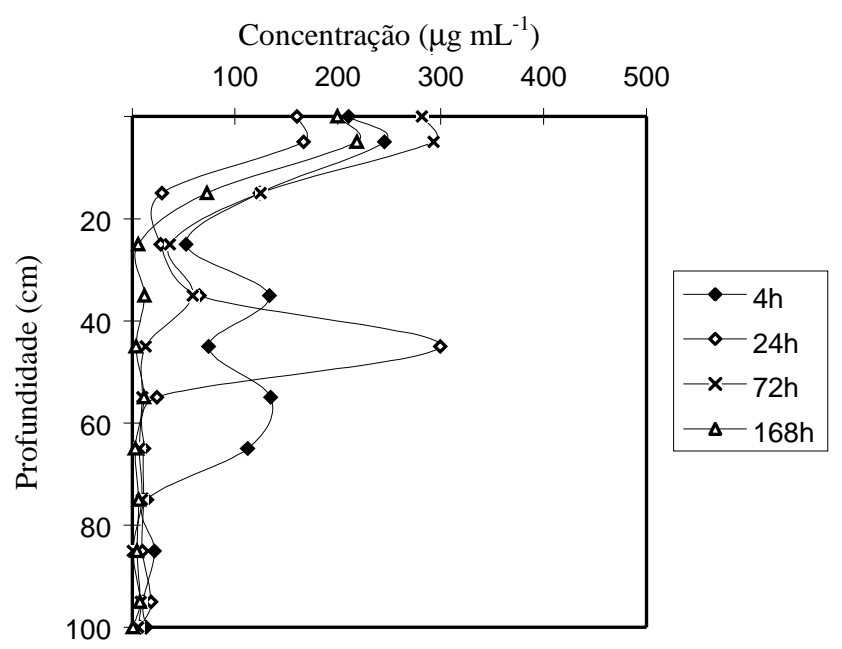

(A) $830 \mathrm{mg} \mathrm{mL}^{-1}$

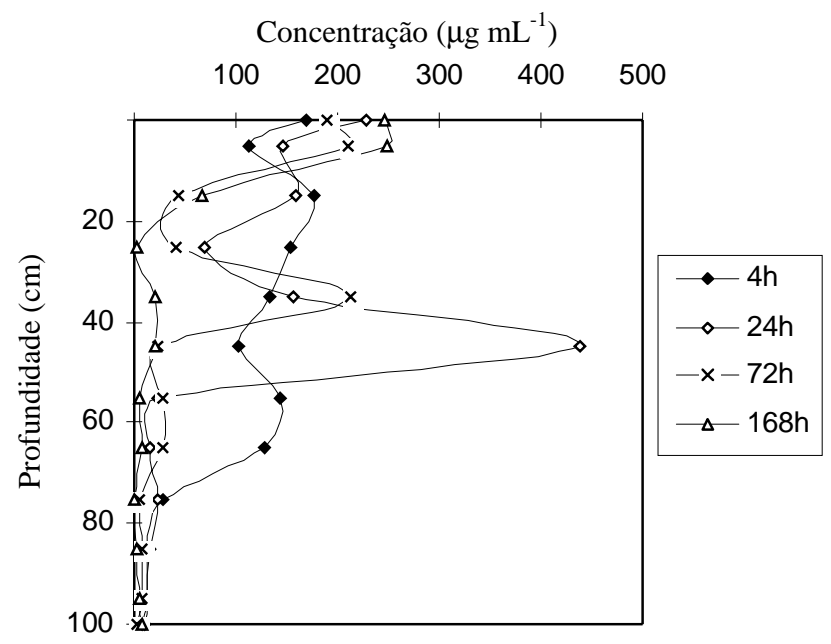

(B) $1.250 \mathrm{mg} \mathrm{mL}^{-1}$

Figura 3. Concentração de nitrato na solução do solo ao longo de seu perfil, em períodos distintos de tempo após sua aplicação, nas concentrações de (a) $830 \mathrm{mg} \mathrm{mL}^{-1} \mathrm{e}$ (b) $1.250 \mathrm{mg} \mathrm{mL}^{-1}$, na superfície superior das colunas

Uma análise das Figuras 2 e 3 indica que os baixos valores de concentração de nitrato nas maiores profundidades são coincidentes com a elevação do conteúdo de água do solo devido à ascensão capilar; com isto, houve diluição do nitrato natural do solo, fazendo baixar sua concentração na solução do solo.

\section{CONCLUSÕES}

O nitrato aplicado ao solo das colunas nas concentrações de 850 e $1.250 \mathrm{mg} \mathrm{mL}^{-1}$ não apresentou grande mobilidade, em decorrência do baixo conteúdo de água no solo. A inexistência do fluxo de massa e a baixa difusão do nitrato impediram que picos de concentração atingissem grandes profundidades, para as duas soluções aplicadas ao solo.

Para avaliar-se o deslocamento de qualquer contaminante em solo não saturado, a determinação da contaminação no solo deve ser preferida, uma vez que independe do conteúdo de água presente. 


\section{REFERÊNCIAS BIBLIOGRÁFICAS}

ALVES, B. Avaliação da mineralização do nitrogênio do solo in situ. Rio de Janeiro: UFRRJ, 1992, p., Dissertação Mestrado EDWARDS, D.M.; FISCHBACH, P.E.; YOUNG, L.L. Movement of nitrate under irrigated agriculture. Transactions of the ASAE, St. Joseph v. , n. , p. 73-75, 1972.

EXNER, M.E.; BURBACH, M.E.; WATTS, D.G.; SHEARMAN, R.C.; SPALDIND, R.F. Deep nitrate movement in the unsaturated zone of simulated urban lawn. Journal Environmental Quality, n. 20, p. 658-662, 1991.

HUBBARD, R.K.; LEONARD, R.A.; JOHNSON, A.W. Nitrate transport on a sandy coastal plain soil underlain by plinthite. Transactions of the ASAE, St. Joseph v.34,n3,p. 802-808, 1991.

MUCHOVEJ, R.M.C.; RECHCIGL, J.E. Impacts of nitrogen fertilization of pastures and turfgrasses on water quality. In: LAL, R.; STEWART, B.A. ed. Soil processes and water quality. Lewis Publication, 1994, p. 91-135.

NEWBOULD, P. The use of nitrogen fertilizer in agriculture. Where do we go practically and ecologically?, Plant Soil, n. 115, p. 297-311, 1989.
NIELSEN, D.R.; BIGGAR, J.W. Miscible displacement in soils: I. Experimental information. Soil Science Society of American Proceeding, Madison, v. 25, n .2, p. 1-5, 1961.

OWENS, L.B. Impacts of soil $\mathrm{N}$ management on the quality of surface and subsurface water. In: LAL, R.; STEWART, B.A. ed. Soil processes and water quality. Lewis Publication, 1994, p. 137-162.

SPALDING, R.F.; EXNER, M.E.; LINDAU, C.W.; EATON, D.W. Investigation of sources of groundwater nitrate contamination in the Burbank Wallula area of Washington, USA. Journal Hydrology, n. 58, p. 307-324. 1982.

VANCLOOSTER, M.; VIAENE, P.; CHRISTIAENS, K. A mathematical model for simulating water and agrochemicals in the soil and vadose environment. Institute for Land and Water Management Leuven: Katholieke Universiteit Leuven, Belgium. 1994.

van GENUCHTEN, M.Th.; WIERENGA, P.J. Mass transfer studies in sorbing porous media. I. Analytical solutions. Soil Science Society of American Proceeding, Madison, v.40, n.3, p.473-480, 1976. 